\title{
Complex Blind Interference Suppression for CDMA System Xin-Quan Luo
}

\author{
Department of science and Engineering,Communication University of China, \\ Beijing,100024,China \\ xqluo@cuc.edu.cn
}

Key words: CDMA system; Interference suppression; Predictive technique; Code-aided technique

\begin{abstract}
Abstrac. To solve the problem that traditional LMS predictive technique is sensitive to complex white Gaussian noise and traditional blind LMS code-aided technique can't reject sinusoidal tone interference in code division multiple access (CDMA) system, a new blind LMS predictive code-aided technique is proposed. The new arithmetic has the ability to suppress complex white Gaussian noise and sinusoidal tone interference at the same time, and is deduced through complex field in this paper. By comparing with LMS predictive and blind LMS code-aided techniques, the advantage of this technique is proved through simulations.
\end{abstract}

\section{Introduction}

The reason of the spread spectrum systems (SSS) being widely used is that it can contrast frequency selective fading caused by multipath as well as its excellent performance in co-channels. The representative core of the technique is CDMA. Although the SSS is equipped with certain anti-interference ability, effective interference suppression technology could observably improve the system performance. In the 21th century, researchers have further developed CDMA system interference suppression techniques into predictive techniques, transform-domain technique and code-aided technique. From all the technologies above, the research of the predictive techniques is concentrated on the improvement of the receiver structure. The specialty of such solution is the simple structure. But it also has typical problem which is high error rate for it processes data bit by bit and fully contrast White Gaussian noise with its own processing gain. With this opinion, code-aided technique, which uses the characteristics of signal code to block processing, seems extremely effective. It is one of the most promising techniques for multi-users system to suppress interferences. In the recent years, blind cod-aided technique is developed to solve the problem that the code-aided technique needs to know the prior knowledge of the interference. Blind-aided technique doesn't need the blind detection of the prior knowledge but it also has the disadvantage that it can't suppress strong narrowband interference.

This paper is going to extend the signal processing from the real- number field to complex-field, learn from the idea of the combination of predictive technique and code-aided technique, put forward a new blind LMS predictive code-aided technique to solve the problem that traditional LMS predictive technique is sensitive to complex white Gaussian noise and traditional blind LMS code-aided technique can't reject sinusoidal tone interference with high power in code division multiple access (CDMA) system. The new arithmetic has the ability to suppress complex white Gaussian noise and sinusoidal tone interference at the same time, and recursion formula is deduced through complex field in this paper.

\section{Signal Model}

\section{Signal Model in time domain}

The envelopment model of the receiving signal when transmitting CDMA signal in non-selective complex white Gaussian noise signal channel with narrowband interference is:

$r(t)=y(t)+i(t)+\varepsilon(t)$

To explain, $y(t)$ represents the CDMA signal, $i(t)$ represents voice interference and $\varepsilon(t)$ 
represents complex white Gaussian noise whose power spectral density (PSD) is $\sigma_{\varepsilon}^{2}$.

In the single synchronous signal channel, CDMA signal model is:

$y(t)=\sum_{k=0}^{K-1} \sqrt{P_{k}} \alpha_{k} \sum_{n=-\infty}^{\infty} b_{k}(n) s_{k}\left(t-n T_{b}\right)$

To explain, $K$ represents the number of effective users; $P_{k}$ represents signal power and $\alpha_{k}$ represents the transferring complex gain with the effect of the channel and $\alpha_{k}=e^{j \phi_{k}}$ in which $\phi_{k}$ is uniformly distributed in $[0,2 \pi] ;\left\{b_{k}(n)\right\}_{n=-\infty}^{+\infty}$ is the signal stream(1 or -1$) ; n$ is the signal tag; $T_{b}$ is the signal symbol period; $s_{k}(t)$ is the sequence spreading the frequency from direct sequence, which is defined as $s_{k}(t)=\frac{1}{\sqrt{N}} \sum_{i=0}^{N-1} c_{k, i} \psi_{c}\left(t-i T_{c}\right)$. In this formula, $\left\{c_{k, i}: i=0, \cdots, N-1\right\}$ is the spreading code(1 or -1$) ; \psi_{c}(\bullet)$ is the normalized waveform of $T_{c}$, the lasting time; $N=T_{b} / T_{c}$ is the spreading gain; $T_{c}$ is the spreading code Chip period.

The voice interference model is:

$i(t)=\sum_{k=0}^{K_{I}-1} \sqrt{P_{I k}} \alpha_{I k} e^{j 2 \pi f_{I k} t}$

To explain, $K_{I}$ is the number of the interferences; $P_{I k}$ is the interference power; $\alpha_{I k}$ is the transferring gain with the effect of the channel and $\alpha_{k}=e^{j \phi_{k}}$ in which $\phi_{k}$ is uniformly distributed in $[0,2 \pi] ; f_{I k}$ is the interference frequency against CDMA carrier frequency. The interference period is set much bigger than the Chip period.

The model could be regarded as multi-voices interference one on the condition that $K_{I}>1$ and single-voice interference one if $K_{I}=1$.

\section{Discrete sampling signal model}

An important solution to solve the problem of interference suppression is to sample the receiving signal $r(t)$ in the infinite dimension linear space and make use of one solution called Chip matched filtering solution. Discrete -time series $r(m)$ is defined as:

$$
r(m)=\frac{1}{T_{c}} \int_{m T_{c}}^{(m+1) T_{c}} r(t) d t=y(m)+i(m)+\varepsilon(m)
$$

\section{CDMA system interference suppression techniques}

\section{LMS prediction technique}

The main idea of the voice interference technique based on prediction is to make use of the differences between narrowband signal and broadband signal and process bit by bit. The biggest advantage of LMS prediction technique is no need of the prior knowledge of the voice interference and useful to suppress the strong voice interference while the biggest disadvantage is to have no effect on broadband interference such as complex white Gaussian noise and multi-site interference, and it just resist to broadband interference with its own spreading gain. Figure 1 tells such interference suppression which is based on LMS prediction.

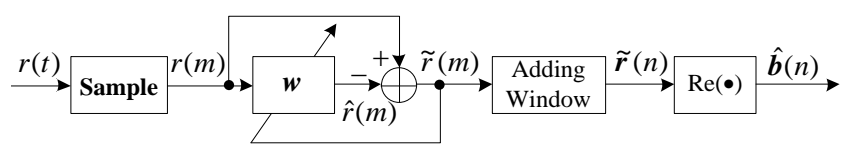

Fig.1. the frame of the interference suppression based on LMS prediction

As what the figure tells, the prediction error, namely the estimate of the CDMA signal is:

$$
\tilde{r}(m)=r(m)-\hat{r}(m)=r(m)-\boldsymbol{w}^{H}(m) \boldsymbol{r}_{M}(m)
$$

In the formula, $\boldsymbol{w}=\left[w_{1}, w_{2}, \cdots, w_{M}\right]^{T}$ is the weight vector and $\boldsymbol{r}_{M}(m)=[r(m-1), r(m-2), \cdots, r(m-M)]^{T}$ is input vector of the filter. 
According to LMS arithmetic, the update equation of the weight vector is:

$$
\boldsymbol{w}(m+1)=\boldsymbol{w}(m)+2 \mu \tilde{r}^{*}(m) \boldsymbol{r}_{M}(m)
$$

To explain, $\mu$ is the step size.

Decode the $\tilde{r}(m)$,you will get the estimate of users signal as follows:

$$
\hat{\boldsymbol{b}}(n)=\operatorname{Re}\left(\boldsymbol{A}^{-1} \boldsymbol{S}^{T} \tilde{\boldsymbol{r}}(n)\right)
$$

To explain, $\tilde{\boldsymbol{r}}(n)=[\tilde{r}(n N+N-1), \tilde{r}(n N+N-2), \cdots, \tilde{r}(n N)]^{T}$ is the vector coming from the sample signal after putting a window on it, CDMA spreading code matrix is $\boldsymbol{S}=\left[s_{0}, s_{1}, \ldots, s_{\mathrm{K}-1}\right]$; and $\operatorname{Re}(\bullet) m e a n s$ the real part of the signal.

Here we can define users mean square error (MSE) average which can reflect signal error rate as:

$$
\operatorname{MSE}(n)=E\left\{\left[\boldsymbol{b}(n)-\operatorname{Re}\left(\boldsymbol{A}^{-1} \boldsymbol{S}^{T} \tilde{\boldsymbol{r}}(n)\right)\right]{ }^{H}\left[\boldsymbol{b}(n)-\operatorname{Re}\left(\boldsymbol{A}^{-1} \boldsymbol{S}^{T} \tilde{\boldsymbol{r}}(n)\right)\right]\right\} / K
$$

\section{Blind LMS code-aided technique}

Blind code-aided arithmetic adopts the method of block processing. Its biggest advantage is to suppress both voice interference and broadband interference and at the meantime, no prior knowledge about the voice interference and no participation of training sequence is required to realize blind detection. However, for it takes the way of approximate evaluation, there exists shortcomings that it is not able to suppress strong voice interference. Figure 2 gives a frame of interference suppression based on blind LMS code-aided technique.

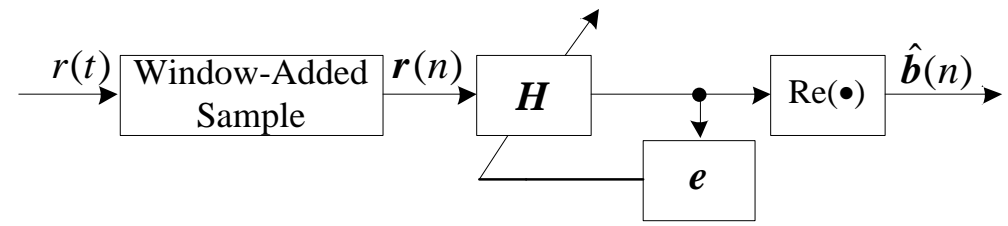

Fig.1. the frame of interference suppression based on blind LMS code-aided technique

Assuming that the adaptive filter coefficient of CDMA is $(N \times K)$ dimension metrix $\boldsymbol{H}$ and error vector is $e(n)=b(n)-\boldsymbol{H}^{H}(n) \boldsymbol{r}(n)$. Take the error vector of the training sequence into the weight coefficient of the LMS code-aided technique update equation, you will get:

$\boldsymbol{H}(n+1)=\boldsymbol{H}(n)+2 \tilde{\mu} \boldsymbol{r}(n)\left[\boldsymbol{b}^{T}(n)-\boldsymbol{r}^{H}(n) \boldsymbol{H}(n)\right]$

To explain, $\tilde{\mu}$ is the LMS code-aided technique step size.

Decode it to get the estimate of the users signal:

$\hat{\boldsymbol{b}}(n)=\operatorname{Re}\left(\boldsymbol{H}^{H}(n) \boldsymbol{r}(n)\right)$

Furthermore, we can get the users MSE average:

$$
\operatorname{MSE}(n)=E\left\{\left[\boldsymbol{b}(n)-\operatorname{Re}\left(\boldsymbol{H}^{H}(n) \boldsymbol{r}(n)\right)\right]^{H}\left[\boldsymbol{b}(n)-\operatorname{Re}\left(\boldsymbol{H}^{H}(n) \boldsymbol{r}(n)\right)\right]\right\} / K
$$

\section{Blind LMS predictive code-aided technique}

Both the voice interference suppression technique based on LMS prediction and the one based on blind LMS code-aided technique have two sides. This section is going to deduce the recurrence formula in blind LMS predictive code-aided technique complex field,which could improve both techniques' defects at the same time. Figure 3 provides an arithmetic frame of the interference suppression based on blind LMS predictive code-aided technique. The blind LMS predictive code-aided interference suppression arithmetic can be divided into two parts, one is LMS predictive filtering and the other is blind LMS code-aided filtering. 


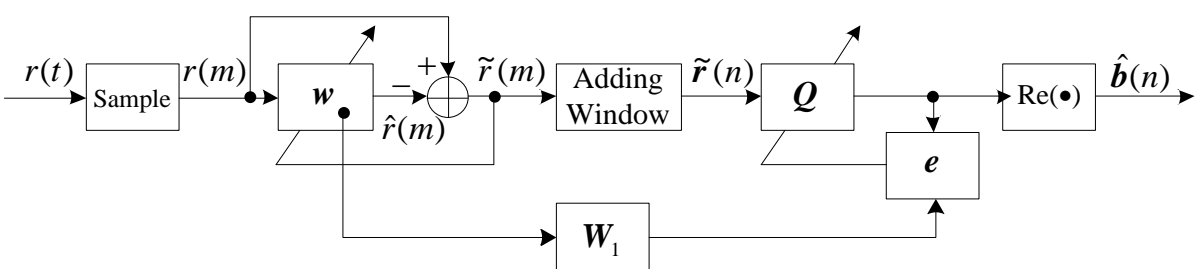

Fig.2. The frame of the interference suppression based on blind LMS predictive code-aided technique

Firstly, make an LMS prediction on the receiving sample signal and the prediction error is:

$\tilde{r}(m)=r(m)-\hat{r}(m)=r(m)-\boldsymbol{w}^{H}(m) \boldsymbol{r}_{M}(m)$

In the formula, weight vector update equation is:

$\boldsymbol{w}(m+1)=\boldsymbol{w}(m)+2 \mu \tilde{\boldsymbol{r}}^{*}(m) \boldsymbol{r}_{M}(m)$

Then, filter the LMS filtering signal with the blind LMS code-aided technique. Assuming there's

a $(N \times K)$ dimension matrix $\boldsymbol{Q}$, the update formula is :

$\boldsymbol{Q}(n+1)=\boldsymbol{Q}(n)+2 \tilde{\mu} \tilde{\boldsymbol{r}}(n) \boldsymbol{e}^{H}(n)$

To explain, $\boldsymbol{e}(n)=\boldsymbol{b}(n)-\boldsymbol{Q}^{H}(n) \tilde{\boldsymbol{r}}(n)$ is the error vector and $\tilde{\mu}$ is the step size.

Decode to get the estimate of the users signal:

$\hat{\boldsymbol{b}}(n)=\operatorname{Re}\left(\boldsymbol{Q}^{H}(n) \tilde{\boldsymbol{r}}(n)\right)$

Furthermore, we can get the users MSE average:

$$
\operatorname{MSE}(n)=E\left\{\left[\boldsymbol{b}(n)-\operatorname{Re}\left(\boldsymbol{Q}^{H}(n) \tilde{\boldsymbol{r}}(n)\right)\right]^{H}\left[\boldsymbol{b}(n)-\operatorname{Re}\left(\boldsymbol{Q}^{H}(n) \tilde{\boldsymbol{r}}(n)\right)\right]\right\} / K
$$

\section{Test results}

\section{Users MSE average in steady state}

In figure 4,(a) and (b) are separately show the curved surface of the users MSE average when the system is in steady state, under the two circumstances, one is single-voice interference while the other is multiple voices(three voices), with the condition that the power ratio of CDMA signal and complex white Gaussian noise(for short, SNR; unit: $\mathrm{dB}$ ) changes synchronously with that of CDMA signal and voice interference(for short, SJR; unit: $\mathrm{dB}$ ). The value is averaged by 100 times. Three conditions are required for simulation: one is CDMA users send the sequence of $1,-1$ with the equal probability and the random sequence whose spreading code $N=63$, one is the frequency of the single-voice interference and three-voices interference is random and another one is step size $\mu$ and $\tilde{\mu}$ is 0.005 . In addition, the 'predictive' in the figure means LMS predictive techniques; code means blind LMS code-aided technique; precode means blind LMS predictive code-aided technique; $J$ means the average of users MSE.

Figure 4 gives a simulation result of effective filtering. It can be seen qualitatively from figure (a) that LMS predictive technique take effect in the case of the strong single-voice interference. The filtering range is rather big $(-30 \mathrm{~dB}<S J R<0 \mathrm{~dB})$, but with the drop of the SNR, the filtering effect worsens obviously and it becomes invalid when $S N R<-20 \mathrm{~dB}$. The LMS code-aided technique exceeds the range of effective filtering when ' $S N R<-20 \mathrm{~dB}$ ' but it's better than LMS predictive technique when $S J R$ is in its rather small effective filtering range. The reason is that it suppresses unitedly complex white Gaussian noise and single-voice interference. It can be seen obviously from the simulated curved surface, the blind LMS predictive code-aided technique proposed in this paper can not only realize effective filtering but also have the marked benefit compared with LMS predictive technique and blind LMS code-aided technique. For multi-voices interference, similar conclusion can be received from figure (b) but specifically, all the arithmetic performances begin to decline for the weaker predictability of multi-voices interference against single-voice interference. To conclude, it is obvious to say that the performance of blind LMS predictive code-aided technique has the absolute advantage over other two techniques. 


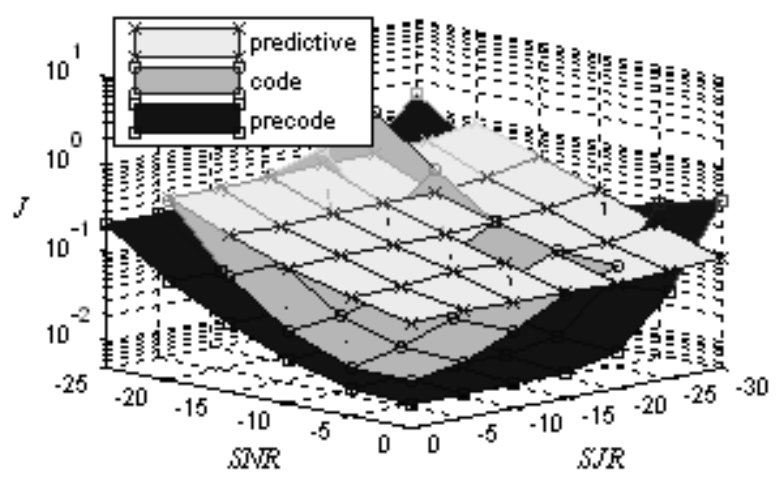

(a)single-voice interference

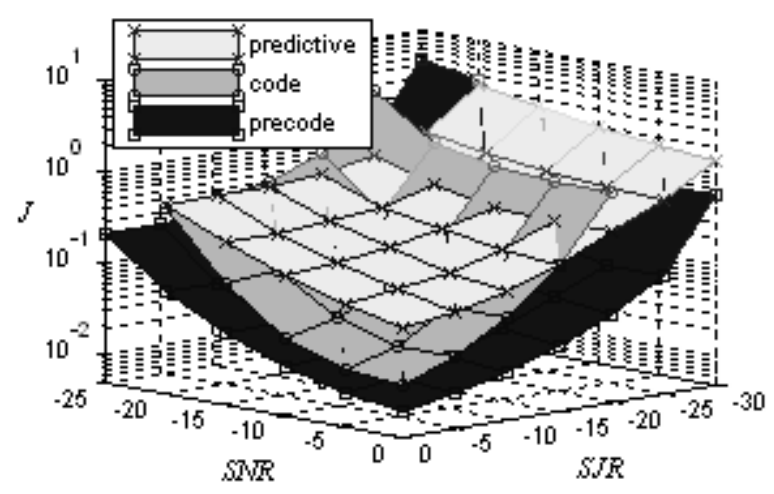

(b)multiple-voices interferences

Fig.3. The curved surface of the users MSE average when the system is in steady state

\section{The convergence performance of the users MSE average}

Figure 5 is the convergence curve of the users MSE average in the case of the single-voice interference. The conditions are: $S N R=-15 \mathrm{~dB}, S J R=-20 \mathrm{~dB}$ and the other ones are the same as those in the last testing section. LMS predictive arithmetic processes signal bit by bit and the filter stages selected are often low. So the convergence speed of bit is fast but nearly shows the direct convergence state when describing the convergence curve of users MSE average. The blind LMS code-aided arithmetic take the method of block processing so the convergence speed is lower-about 500 steps can lead to convergence under such simulation conditions. The blind LMS predictive code-aided arithmetic is effected by both the convergence speeds of LMS predictive and blind LMS code-aided techniques. Its convergence speed is between them- about 250 steps is enough, and it is largely controlled by the blind LMS code-aided arithmetic or the LMS predictive arithmetic has an extremely fast convergence speed. In addition, it can be seen quantificationally that blind LMS predictive code-aided arithmetic has the absolute advantage.

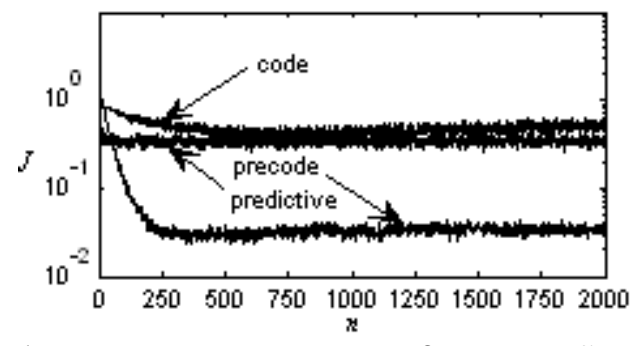

Fig.4. The convergence curve of users MSE average

\section{Conclusion}

This paper learns from the idea of combining predictive technique and code assist technique, extends the signal processing from the real number field to complex number field, puts forward a detection solution to use the brand new blind LMS predictive code-aided technique to suppress the interference of the CDMA system and deduces the recursion formula of blind LMS predictive code-aided arithmetic in the complex field. The solution not only solves the problem of the current LMS predictive techniques not being able to suppress complex white Gaussian noise and the blind LMS code-aided technique not being able to suppress the interference of the strong voices but also realizes a blind detection which is not required the prior knowledge of voice interference. The simulation firstly analyzes users MSE average in steady state to prove its advantage over LMS predictive and blind LMS code assist arithmetics and make the further analysis on the convergence performance, leading to the conclusion that the convergence speed falls in between. 


\section{References}

[1] L B Milstein. Interference rejection techniques in spread spectrum communication[J]. IEEE Proceedings. 1988, Vol.76:657-667P

[2] H Vincent, L A Rusch. Narrowband interference suppression in spread spectrum CDMA[J]. IEEE Personal Communication. 1994, Vol.3:14-27P

[3] Xiaodong Wang, H V Poor. Translated by Baoyu Zheng etc. Wireless Communication Systems: Advanced Techniques for Signal Reception[M]. Edition 1. Beijing:Publishing House of Electronics Industry, 2005: 289-334P

[4] Sun Yongjun,Yi Kechu. Suppression of multiple narrowband interference using kernel methods in DSSS[J]. Journal of Xidian University. 2007, Vol.34:554-558P

[5] Benjebbour Anass, Asai Takahiro, Yoshino Hitoshi. Nonparametric interference suppression using cyclic wiener filtering: pulse shape design and performance evaluation[J]. Eurasip Journal on Wireless Communications. 2008, Vol.1:243-248P

[6] H V Poor. Active interference suppression in CDMA overlay systems[J]. IEEE Journal. on Selected. Areas. in Communications. 2001, Vol.19:4-20P

[7] S Buzzi, M Lops, H V Poor. Code-aided interference suppression for DS/CDMA overlay systems[J]. IEEE Proceedings. 2002, Vol.90:394-435P

[8] S Buzzi, A M De, M Lops. Code-aided blind adaptive new user detection in DS/CDMA systems with fading time-dispersive channels[J]. IEEE Trans. Signal. Pro. 2003, Vol.51:2637-2649P [9] Xiaoning Lu, K C Ho. Novel adaptive methods for narrowband interference cancellation in CDMA multi-user detection[J]. IEEE ICASSP. 2005, Vol.3:921-924P

[10] K C Ho, Xiaoning Lu, Vandana Mehta. Adaptive blind narrowband interference cancellation for multi-user detection[J]. IEEE Transaction. on Wireless communications. 2007, Vol.3:1024-1033P 